\title{
Mesenchymal stem cells enhance NOX2-dependent reactive oxygen species production and bacterial killing in macrophages during sepsis
}

\author{
Razieh Rabani ${ }^{1,9}$, Allen Volchuk ${ }^{1,9}$, Mirjana Jerkic ${ }^{1}$, Lindsay Ormesher ${ }^{1}$, \\ Linda Garces-Ramirez ${ }^{1,2}$, Johnathan Canton ${ }^{3}$, Claire Masterson ${ }^{1}$, \\ Stephane Gagnon ${ }^{1}$, Kate C. Tatham ${ }^{1,4}$, John Marshall ${ }^{1,5}$, Sergio Grinstein ${ }^{3}$, \\ John G. Laffey (10 ${ }^{1,6,7}$, Katalin Szaszi $i^{1,5,10}$ and Gerard F. Curley (iD) 1,7,8,10
}

Affiliations: ${ }^{1}$ Critical Illness and Injury Research Centre, Keenan Research Centre for Biomedical Science of St Michael's Hospital, Toronto, ON, Canada. ${ }^{2}$ Dept of Physiology, Escuela Nacional de Ciencias Biologicas, Mexico City, Mexico. ${ }^{3}$ Cell Biology Program, The Hospital for Sick Children, Toronto, ON, Canada. ${ }^{4}$ Section of Anaesthetics, Pain Medicine and Intensive Care, Dept of Surgery and Cancer, Imperial College London, London, UK. ${ }^{5}$ Dept of Surgery, University of Toronto, Toronto, ON, Canada. ${ }^{6}$ Dept of Physiology, University of Toronto, Toronto, ON, Canada. ${ }^{7}$ Dept of Anesthesia, University of Toronto, Toronto, ON, Canada. ${ }^{8}$ Dept of Anaesthesia and Critical Care, Royal College of Surgeons in Ireland, Dublin, Ireland. ${ }^{9}$ These two authors contributed equally to this work. ${ }^{10}$ These two authors contributed equally to this work.

Correspondence: Gerard F. Curley, Royal College of Surgeons in Ireland Education and Research Centre, Beaumont Hospital, Dublin, D09 YD60, Ireland. E-mail: gercurleyarcsi.ie

@ERSpublications

Mesenchymal stem cells enhance bacterial killing via production of phagosomal reactive oxygen species in macrophages http://ow.ly/tigl30iGFPG

Cite this article as: Rabani $\mathrm{R}$, Volchuk $\mathrm{A}$, Jerkic $\mathrm{M}$, et al. Mesenchymal stem cells enhance NOX2-dependent reactive oxygen species production and bacterial killing in macrophages during sepsis. Eur Respir J 2018; 51: 1702021 [https://doi.org/10.1183/13993003.02021-2017].

ABSTRACT Human mesenchymal stem/stromal cells (MSCs) have been reported to produce an M2-like, alternatively activated phenotype in macrophages. In addition, MSCs mediate effective bacterial clearance in pre-clinical sepsis models. Thus, MSCs have a paradoxical antimicrobial and anti-inflammatory response that is not understood.

Here, we studied the phenotypic and functional response of monocyte-derived human macrophages to MSC exposure in vitro.

MSCs induced two distinct, coexistent phenotypes: M2-like macrophages (generally elongated morphology, $\mathrm{CD}_{163}{ }^{+}$, acute phagosomal acidification, low NOX2 expression and limited phagosomal superoxide production) and M1-like macrophages characterised by high levels of phagosomal superoxide production. Enhanced phagosomal reactive oxygen species production was also observed in alveolar macrophages from a rodent model of pneumonia-induced sepsis. The production of M1-like macrophages was dependent on prostaglandin $\mathrm{E}_{2}$ and phosphatidylinositol 3-kinase. MSCs enhanced human macrophage phagocytosis of unopsonised bacteria and enhanced bacterial killing compared with untreated macrophages. Bacterial killing was significantly reduced by blockade of NOX2 using diphenyleneiodonium, suggesting that M1-like cells are primarily responsible for this effect. MSCs also enhanced phagocytosis and polarisation of M1-like macrophages derived from patients with severe sepsis.

The enhanced antimicrobial capacity (M1-like) and inflammation resolving phenotype (M2-like) may account for the paradoxical effect of these cells in sepsis in vivo. 


\section{Introduction}

Sepsis is the biggest cause of mortality in critically ill patients and may contribute to half of all hospital deaths $[1,2]$. Improved treatment of sepsis could offer meaningful improvements in population mortality.

Mesenchymal stem/stromal cells (MSCs) are stromal cells that form a supportive, perivascular niche for haematopoietic stem cells (HSCs) in bone marrow and coordinate the trafficking of HSCs and monocytes [3, 4]. Although initially identified in bone marrow [5], MSCs are also present as perivascular cells in other tissues, including muscle, umbilical cord and adipose tissue [6], and are easily isolated and expanded in culture. MSCs have demonstrated promise in pre-clinical models [7-9] such that they are being tested in early-phase clinical trials in critically ill patients with sepsis $[10,11]$.

A key interaction for the therapeutic benefit of MSCs is that of MSCs and monocytes/macrophages. MSCs have been shown to alter the behaviour of mononuclear phagocytes to induce an anti-inflammatory (M2) or tolerant phenotype [12]. MSCs facilitate monocyte-to-macrophage transition, attenuate activated M1 macrophages and enhance M2 activation [13]. This MSC-mediated modulation of monocyte/macrophage immune responses is believed to play a critical role in their therapeutic effects [14]. M2 macrophages suppress inflammation and facilitate wound repair, but have limited cellular machinery capable of effective microbicidal responses, including the powerful oxidative system composed of the NADPH oxidase [15]. Interleukin (IL)-10-secreting M2 macrophages are particularly adept at suppressing antimicrobial immunity, and can enhance the survival and spread of some microorganisms [16, 17]. However, this anti-inflammatory phenotype is inconsistent, with several studies demonstrating MSCs to be effective in improving bacterial clearance and survival in animal models of sepsis, an effect that appears dependent in part on macrophages $[14,18-20]$.

Thus, it remains unclear how MSCs can both enhance bacterial clearance and induce an anti-inflammatory macrophage phenotype.

To better define the effect of MSCs on macrophages, we studied human macrophage phenotype and function in response to human bone marrow-derived MSC exposure. Specifically, we wished to define how exposure to MSCs alters macrophage polarisation and key functions such as superoxide production and phagocytosis. Finally, we also aimed to identify some of the mediators and signalling pathways through which MSCs alter macrophage functions.

\section{Materials and methods}

All work was approved by the Human Ethics Committee and the Animal Care and Use Committee of the Keenan Research Centre for Biomedical Science of St Michael's Hospital (Toronto, ON, Canada), and conducted under licence from Health Canada. Detailed methods are described in the supplementary material.

\section{MSCs, fibroblasts and monocyte/macrophage isolation and culture}

Human bone marrow MSCs were obtained from the Center for the Preparation and Distribution of Adult Stem Cells (medicine.tamhsc.edu/irm/msc-distribution.html) at Texas A\&M Health Science Center College of Medicine Institute for Regenerative Medicine (Temple, TX, USA). Human umbilical cord MSCs were provided by Tissue Regeneration Therapeutics (Toronto, ON, Canada). The cells fulfilled international criteria. Human normal dermal fibroblasts were purchased from Lonza (Mississauga, ON, Canada). Human peripheral blood mononuclear cells were isolated from the blood of healthy adult donors or patients with sepsis using dextran/Ficoll or Lympholyte $\mathrm{H}$ cell separation media (CL5015; Cedarlane, Burlington, ON, Canada). Detailed protocols are provided in the supplementary material. Briefly, following isolation, human blood-derived monocytes were cultured for 8 days with media containing serum (defined as M0 macrophages) or after 5 days the cells were cultured with bone marrow-derived MSCs in transwell or coculture for 3 days as indicated in the figure legends. For production of defined M1 or M2 macrophages, monocytes were treated with macrophage colony-stimulating factor $\left(25 \mathrm{ng} \cdot \mathrm{mL}^{-1}\right)$ for 6 days then IL-4 $\left(10 \mathrm{ng} \cdot \mathrm{mL}^{-1}\right)$ for 2 days (M2), or granulocyte-macrophage colony-stimulating factor $\left(25 \mathrm{ng} \cdot \mathrm{mL}^{-1}\right)$ for 6 days then interferon- $\gamma\left(10 \mathrm{ng} \cdot \mathrm{mL}^{-1}\right)$ and lipopolysaccharide $\left(500 \mathrm{ng} \cdot \mathrm{mL}^{-1}\right)$ for 2 days (M1), as described previously [15].

\section{Serum opsonised zymosan and nitroblue tetrazolium assay}

Dried Zymosan A (Saccharomyces cerevisiae) Bioparticles (Life Technologies, Burlington, ON, Canada) were labelled with fluorophores (Alexa-555 or fluorescein isothiocyanate (FITC)) as described previously [15].

This article has supplementary material available from erj.ersjournals.com

Received: Oct 032017 | Accepted after revision: Feb 212018 
Nitroblue tetrazolium (NBT) assays were performed as described previously [15]. Briefly, Alexa-555conjugated serum opsonised zymosan (SOZ) and NBT (N5514-25TAB; Sigma, Oakville, ON, Canada) were added to cells at the concentrations indicated in the figure legends for $30 \mathrm{~min}$, washed and then fixed in $4 \%$ paraformaldehyde in PBS for $15 \mathrm{~min}$. Cells were visualised by differential interference contrast microscopy. Dark formazan deposits were indicative of phagosomal superoxide production. In some experiments, after the NBT assay, the cells were fixed in ice-cold methanol for $2 \mathrm{~min}$ at $-20^{\circ} \mathrm{C}$ and processed for immunofluorescence microscopy.

\section{Bacterial phagocytosis, phagosomal pH measurements and killing assay}

Cultures of Red Fluorescent Protein (RFP)-expressing Escherichia coli (DH5a) or RFP-expressing Burkholderia cenocepacia were added to macrophage cells at the concentration indicated in the figure legends, washed and fixed in $4 \%$ paraformaldehyde/bovine serum albumin for $15 \mathrm{~min}$. Bacterial phagocytosis was monitored by spinning disc confocal microscopy. Phagosomal $\mathrm{pH}$ was measured using ratio imaging as described previously [15]. For bacterial killing, the gentamicin exclusion assay was used, as described in the supplementary material.

\section{Rodent E. coli-induced injury}

Specific pathogen-free adult male Sprague Dawley rats (Charles River Laboratories, Quebec, QC, Canada) weighing 350-450 g were used in all experiments. Animals were anaesthetised and intubated with a $14 \mathrm{G}$ intravenous catheter (BD Insyte; Becton Dickinson, Oxford, UK). $2 \times 10^{9}$ CFU of E. coli in a $300 \mu \mathrm{L}$ PBS suspension was instilled into the trachea using a $1 \mathrm{~mL}$ syringe, and the animals allowed to recover and treated with $10 \times 10^{6} \mathrm{MSCs} \cdot \mathrm{kg}^{-1}$ or PBS [21]. After $48 \mathrm{~h}$ the animals were killed by exsanguination under anaesthesia. The heart-lung block was dissected from the thorax, bronchoalveolar lavage was performed and alveolar macrophages were isolated using Lympholyte R cell separation media (CL5041; Cedarlane).

\section{Statistical analysis}

All values reported are mean with standard deviation or standard error as stated in the figure legends. An unpaired t-test assuming equal variance was used for two-condition comparisons. ANOVA followed by Tukey's post hoc test was used for multiple-condition comparisons. $\mathrm{p}<0.05$ was considered significant.

\section{Results}

MSCs promote production of phagosomal reactive oxygen species in macrophages in vitro and in vivo MSCs have been reported to enhance bacterial clearance in several animal models of infection in vivo, although the precise mechanism remains unclear [14, 18-20, 22]. Mitochondrial transfer [22, 23] and production of antimicrobial peptides (LL-37 [24] and lipocalin 2 [25]) have been shown to be involved in bacterial clearance by MSCs. Moreover, M1 macrophages in particular are thought to have potent antimicrobial capacity in part due to high levels and activity of the phagosomal NADPH oxidase that facilitates bacterial killing [15]. To examine the effect of MSCs on macrophage phagosomal oxidase activity, human bone marrow-derived MSCs were cocultured with human monocyte-derived macrophages and phagosomal superoxide was monitored by exposing macrophages to SOZ in the presence of NBT. The product of NBT reacting with superoxide is formazan, a dark deposit easily visualised by light microscopy [15].

Remarkably, as shown in figure $1 \mathrm{a}$ and $\mathrm{b}$, under conditions where limited or no phagosomal superoxide was detected in control M0 cells (white arrows), many formazan-positive cells were apparent in M0 cells that were exposed to MSCs (figure 1a, yellow arrows). This M1-like phenotype was observed in both transwell and coculture conditions. M0 cells exposed to fibroblasts rather than MSCs demonstrated no change in production of superoxide (supplementary figure S1). Formazan production was dependent on NADPH oxidase activity as no cells with phagosomal formazan deposits were observed in M0+MSC cells treated with the NADPH oxidase inhibitor diphenyleneiodonium (DPI) (figure 1a), which did not affect the ability of the cells to phagocytose SOZ. Overall, under the conditions of the experiment, there was $\sim 3$-fold increase in formazan-positive macrophages exposed to MSCs in transwell compared with M0 control (figure 1b). Additionally, we observed that at high NBT concentrations, where now most of the round cells produced phagosomal formazan (figure 1c, yellow arrows), a subset of mostly elongated macrophages in the M0+MSC condition was completely devoid of phagosomal formazan (figure 1c, inset, white arrows). This is indicative of very limited phagosomal superoxide production, which is a feature of M2 macrophages (figure 1c, right panel) [15]. To verify that macrophage phagosomal oxidase activity is indeed enhanced by MSC exposure we performed a luminol assay in live macrophages (figure $2 a$ and $b$ ). Analysis of multiple experiments revealed a significant increase in phagosomal reactive oxygen species (ROS) production at early time-points in macrophage cells exposed to MSCs (figure $2 \mathrm{~b}$ ).

In order to determine whether enhanced phagosomal superoxide production in response to MSCs occurs in vivo, we isolated macrophages from a rodent model of E. coli pneumonia-induced sepsis. Alveolar 
a)
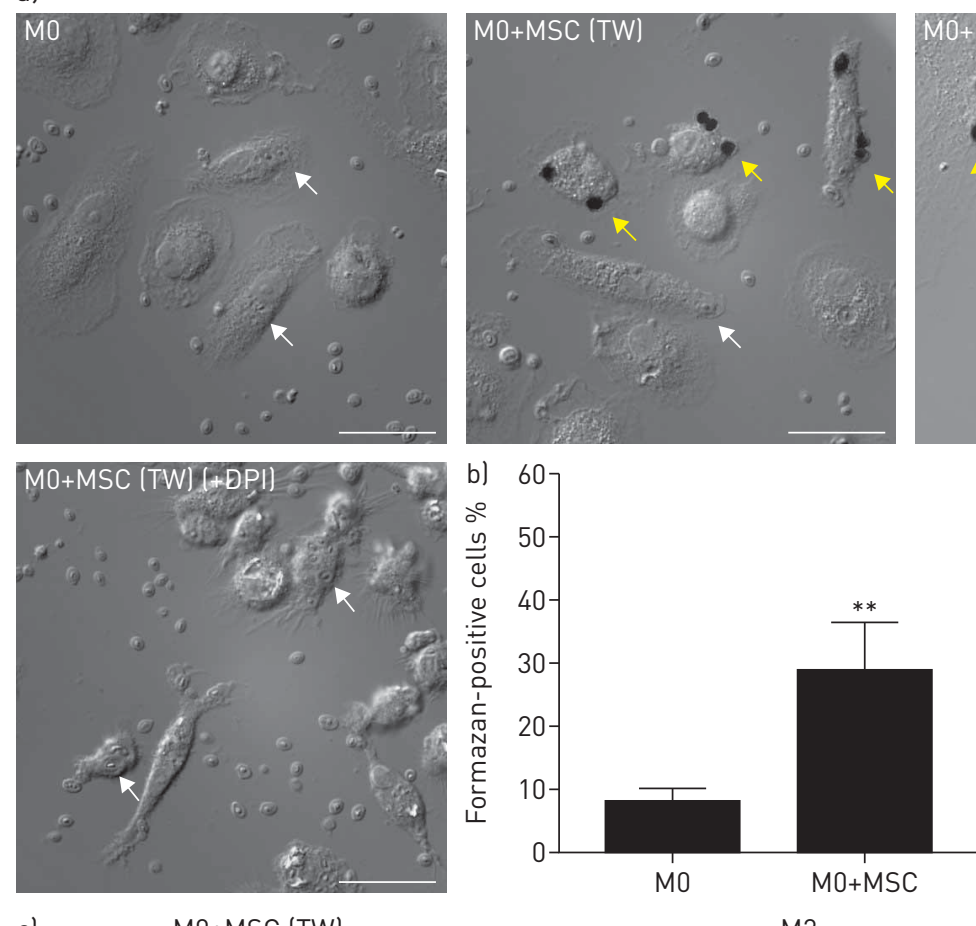
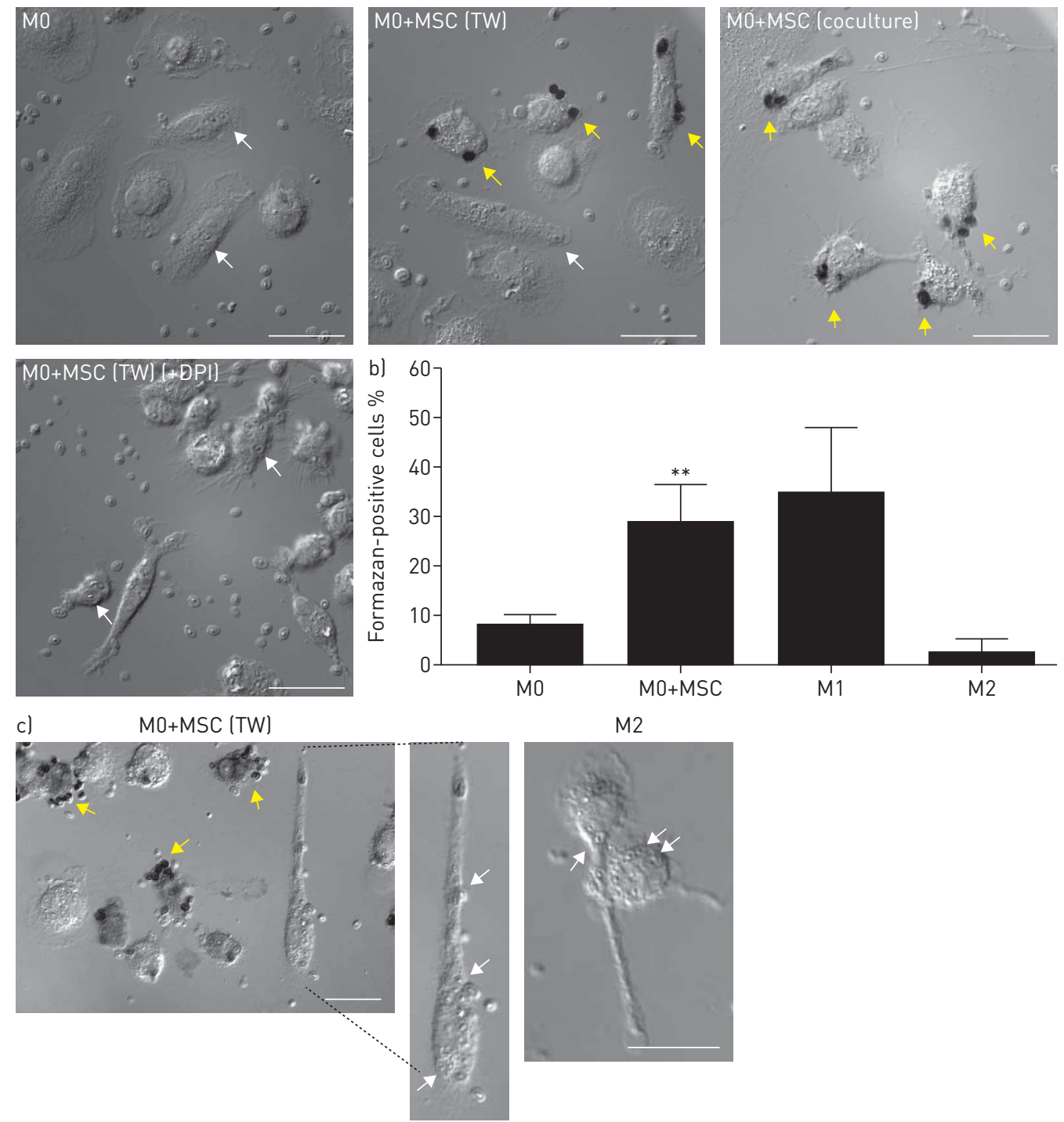

FIGURE 1 Effect of human mesenchymal stem/stromal cells (MSCs) on phagosomal reactive oxygen species production in human macrophages (nitroblue tetrazolium (NBT) assay). a) Human blood-derived monocytes were cultured for 8 days with media containing serum $(\mathrm{MO})$ or after 5 days the cells were cultured with bone marrow-derived MSCs in transwell (TW) or coculture for 3 days. The cells were then incubated with $0.01 \mathrm{mg} \cdot \mathrm{mL}^{-1}$ serum opsonised zymosan (SOZ) together with $0.125 \mathrm{mg} \cdot \mathrm{mL}^{-1} \mathrm{NBT}$ in the absence or presence of $10 \mu \mathrm{M}$ diphenyleneiodonium (DPI), as indicated. The cells were fixed and imaged by differential interference contrast (DIC) microscopy. White arrows indicate cells that have phagocytosed SOZ that are devoid of formazan deposits. With MSC exposure numerous cells had clear evidence of phagosomal formazan, indicative of high phagosomal superoxide production (yellow arrows). Representative images are shown for the various conditions. Scale bar: $20 \mu \mathrm{m}$. b) Quantitation of formazan-positive cells. Data are presented as mean \pm SD. $n=4$ independent experiments. ${ }^{* *}: \mathrm{p}<0.01$, MO versus M0+MSC (TW). c) M0 cells exposed to MSCs (TW) or M2 cells were incubated with SOZ and $2 \mathrm{mg} \cdot \mathrm{mL}^{-1}$ NBT for $30 \mathrm{~min}$. The cells were then washed, fixed and imaged by DIC microscopy. Scale bar: $20 \mu \mathrm{m}$. Two phenotypes were observed: most round cells that phagocytosed SOZ had dark formazan deposits (yellow arrows in left panel), while elongated cells that phagocytosed SOZ had limited or no formazan deposits in phagosomes (white arrows in inset), similar to M2 cells (right panel).

macrophages were isolated after treatment of rats with MSCs or PBS, and an NBT assay was performed after isolation and culture. The number of formazan-positive cells (indicative of high phagosomal superoxide production) was significantly increased in the MSC-treated condition (figure 3).

MSC-induced phagosomal ROS production in macrophages is dependent on activation of NOX2 Given that phagosomal superoxide production is dependent on the NADPH oxidase enzyme, we examined the expression and localisation of gp $91^{\text {phox }}$, the membrane-bound catalytic subunit of the NOX2 isoform, 

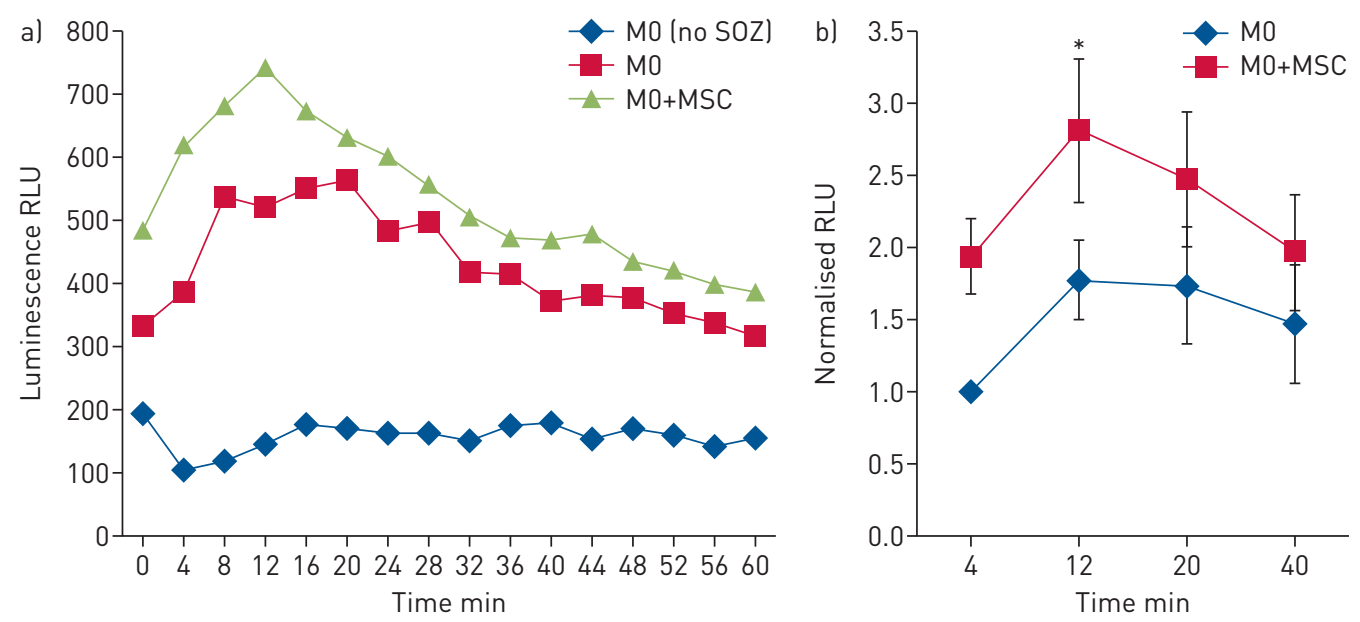

FIGURE 2 Effect of human mesenchymal stem/stromal cells (MSCs) on phagosomal reactive oxygen species (ROS) production in human macrophages (luminol assay). Human blood-derived monocytes were seeded in a 96-well plate, cultured for 8 days with media containing serum (M0) or after 5 days the cells were cocultured with bone marrow-derived MSCs for 3 days. A luminol assay was performed to monitor phagosomal ROS production as described in the Materials and methods. Luminescence in relative luminescence units (RLU) was monitored over time. SOZ: serum opsonised zymosan. a) Representative single experiment showing control macrophages not exposed to SOZ, or control or MSC-exposed macrophages treated with SOZ. An increase in luminescence is only observed in macrophages exposed to SOZ. b) Data normalised to RLU value at $\mathrm{t}=4$ min. $\mathrm{n}=5$ independent experiments. ${ }^{*}: \mathrm{p}<0.05$.

which has been shown to be expressed at higher levels in M1 compared with M2 macrophages [15]. gp91 ${ }^{\text {phox }}$ expression was readily detected in both control M0 cells, as well as in macrophage cells in the M0+MSC condition (supplementary figure S2, white arrows). In contrast, elongated M2-like cells observed only in the M0+MSC condition had low gp91 ${ }^{\text {phox }}$ expression (supplementary figure S2, yellow arrows). gp91 ${ }^{\text {phox }}$ staining in macrophage cells following SOZ phagocytosis in the NBT assay revealed that expression is highest in cells that produce phagosomal superoxide as detected by formazan production (supplementary figure S2, white arrowheads), while M2-like cells had lower levels of gp9 ${ }^{\text {phox }}$. However, total gp91 ${ }^{\text {phox }}$ levels in round macrophage cells in the M0+MSC condition were not significantly different from control M0 cells (supplementary figure S2d and e).

Thus, enhanced phagocytosis-induced NADPH oxidase activation, rather than increased levels of the catalytic NOX2 subunit, is the more likely mechanism for MSC-induced ROS production in macrophages. Phagocytosis-induced activation of the oxidase is a complex and highly regulated process that involves assembly of a functional multisubunit enzyme complex at the newly forming phagosome by recruitment of several cytosolic subunits to the membrane-bound components gp91 phox and $\mathrm{p} 22^{\text {phox }}[26,27]$. We examined the localisation of one of the cytosolic subunits, p47 $7^{\text {phox }}$. An NBT assay was performed in M0

a)

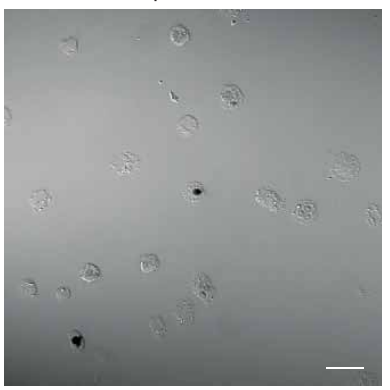

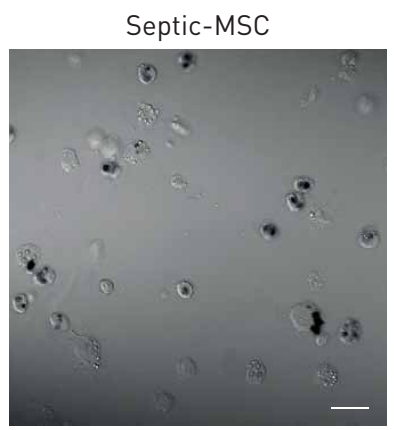

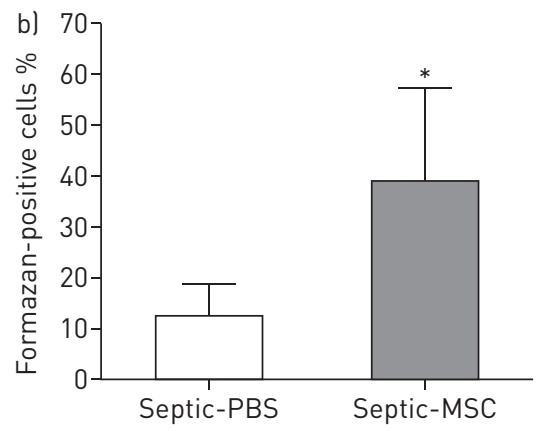

FIGURE 3 Effect of human mesenchymal stem/stromal cells (MSCs) on phagosomal reactive oxygen species (ROS) production in isolated rat alveolar macrophages from an Escherichia coli pneumonia model. Alveolar macrophages were isolated from rats exposed to E. coli pneumonia, then treated with either PBS or MSCs for $48 \mathrm{~h}$ as described in the Materials and methods. A serum opsonised zymosan-nitroblue tetrazolium assay was then performed. a) Representative images. Phagosomal formazan deposits indicative of phagosomal ROS appear black. Scale bar: $20 \mu \mathrm{m}$. b) Quantitation of formazan-positive cells. Data are presented as mean \pm SD. $n=4$ experiments. ${ }^{*}: p<0.05$. 
p47phox (red)
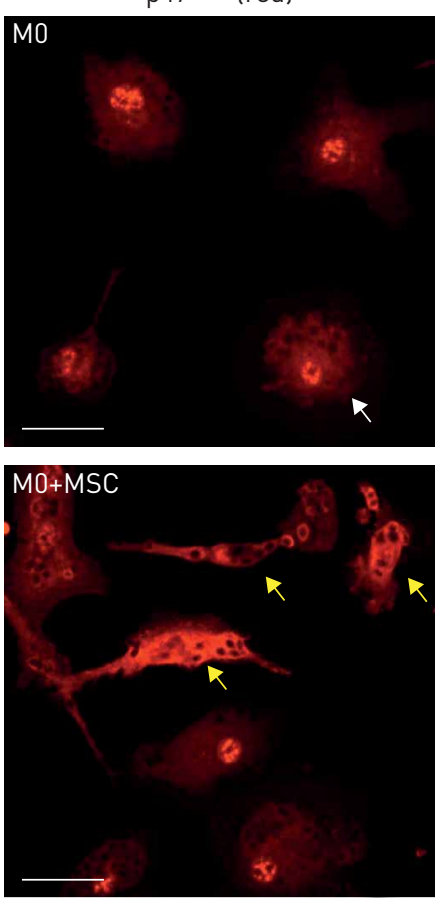

SOZ (green)
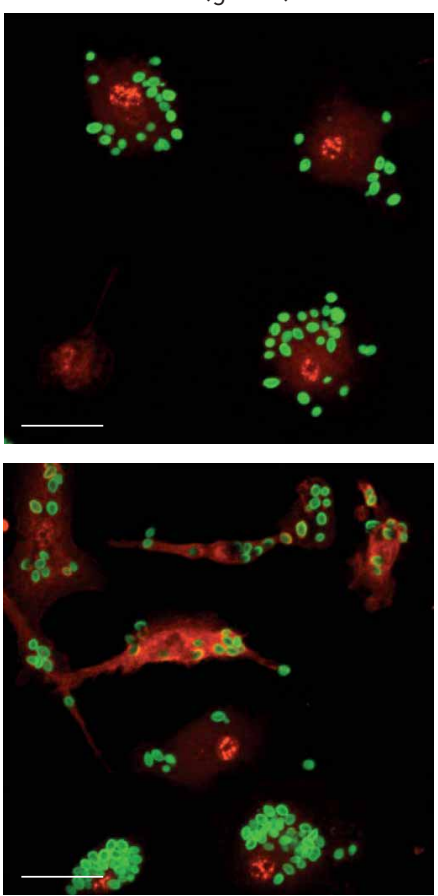

Formazan (black)
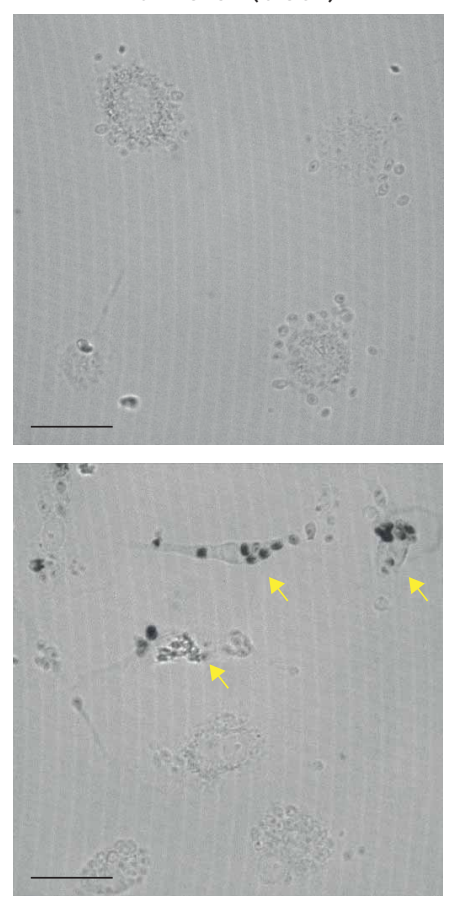

FIGURE 4 M1-like macrophages have increased phagosomal levels of the NADPH oxidase subunit p47 ${ }^{\text {phox }}$ Human macrophages were exposed or not to bone marrow-derived mesenchymal stem/stromal cells (MSCs) in transwell for 3 days. The cells were then incubated with serum opsonised zymosan together with $0.125 \mathrm{mg} \cdot \mathrm{mL}^{-1}$ nitroblue tetrazolium for $30 \mathrm{~min}$. The cells were fixed and immunostained for $\mathrm{p} 47^{\text {phox }}$. In control MO cells with no detectable phagosomal superoxide production, $\mathrm{p} 47^{\text {phox }}$ immunostaining was apparent in a clustered perinuclear region in the cytosol and weak staining was observed in some cells surrounding internalised zymosan particles (white arrow). In MO exposed to $\mathrm{MSCs}$ (MO+MSC) many cells producing abundant phagosomal superoxide, as detected by dark formazan deposits, were evident lyellow arrows in bottom right panel). In general these cells had higher levels of $\mathrm{p} 47^{\text {phox }}$ immunostaining surrounding internalised zymosan particles (yellow arrows in bottom left panel). SOZ: serum opsonised zymosan. Scale bar: $10 \mu \mathrm{m}$.

cells or M0 exposed to MSCs, followed by immunostaining for $447^{\text {phox }}$. As shown in figure 4, most M0 cells that phagocytosed SOZ did not produce formazan under the assay conditions used, while most of the $\mathrm{p} 47^{\text {phox }}$ was cytosolic or sequestered in a perinuclear location. In M0 cells exposed to MSCs, phagosomal ROS production was evident as revealed by dark phagosomal formazan deposits (figure 4, bottom right panel, yellow arrows). In these cells many of the zymosan particles were surrounded by bright $\mathrm{p} 47^{\text {phox }}$ immunostaining (figure 4, bottom left panel, yellow arrows). Thus, cells with enhanced ability to produce phagosomal superoxide, which are more prevalent in the M0+MSC condition, have overall increased levels of active oxidase.

\section{MSC-induced phagosomal ROS production is dependent on prostaglandin $E_{2}$ and phosphatidylinositol 3-kinase}

Next, we wished to gain insight into the mechanisms whereby MSCs augment oxidase activity. In response to phagocytosis, the cytosolic subunits $\mathrm{p} 47^{\text {phox }}$ and $\mathrm{p} 40^{\text {phox }}$ are recruited to the phagosome by binding to phosphatidylinositol 3,4-bisphosphate and phosphatidylinositol 3-phosphate, respectively, which are generated by a distinct phosphatidylinositol 3-kinase (PI3K) [26-28]. Thus, we hypothesised that PI3K activity might be increased in some M0 cells exposed to MSCs and that this may in part contribute to the enhanced ability to recruit the cytosolic oxidase subunits, resulting in enhanced ability to activate the oxidase in response to a phagocytic stimulus. Analysis of phospho-Akt, a downstream target of the PI3K pathway, showed an increase in M0 cells exposed to MSCs compared with M0 alone (figure 5a). Furthermore, inhibition of PI3K activity with wortmannin prevented the increase in cells with enhanced phagosomal superoxide production (figure 5b) without affecting SOZ phagocytosis (figure 5c), indicating that PI3K activity is required for this effect. Similar results were obtained with the PI3K inhibitor LY294002 (results not shown).

PI3K can be activated by a number of factors including prostaglandin $\mathrm{E}_{2}\left(\mathrm{PGE}_{2}\right)$, which is known to be produced by MSCs [14]. Indeed, $\mathrm{PGE}_{2}$ alone enhances phospho-Akt levels in human M0 macrophages (figure 5d) and $\mathrm{PGE}_{2}$ levels are markedly enhanced when MSCs are cocultured with macrophages (figure 5e). 

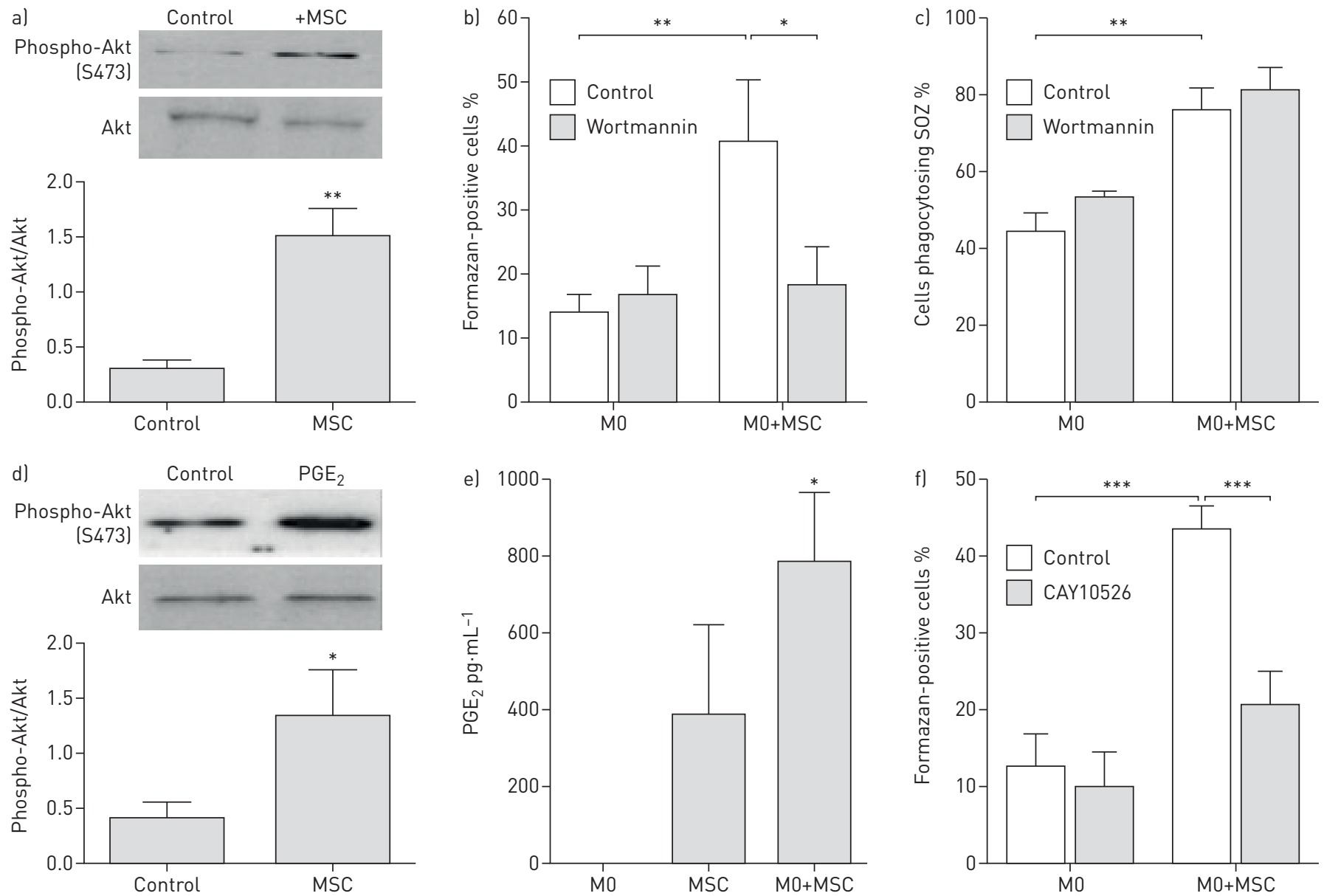

FIGURE 5 Mesenchymal stem/stromal cell (MSC)-induced production of $M 1$-like macrophages requires prostaglandin $E_{2}$ (PGE $E_{2}$ and phosphatidylinositol 3-kinase (PI3K). Human blood-derived monocytes were cultured for 8 days with media containing serum (M0) or after 5 days the cells were cultured with bone marrow-derived MSCs in transwell for 3 days. a) To monitor PI3K activation in the macrophages exposed to MSCs, total cell lysates were subjected to Western blotting with anti-phospho-Akt (S473). To normalise for protein loading, the membrane was probed with anti-total Akt. Data are presented as mean \pm SD. $n=3$. ${ }^{* *}: p<0.01$. b, c) To confirm the role of PI3K activation, M0 cells were cultured with MSCs in transwell in the absence or presence of $1 \mu \mathrm{M}$ wortmannin PI3K inhibitor. Cells were then incubated with $0.01 \mathrm{mg} \cdot \mathrm{mL}^{-1}$ serum opsonised zymosan (SOZ) together with $0.125 \mathrm{mg} \cdot \mathrm{mL}^{-1}$ nitroblue tetrazolium (NBT) for $30 \mathrm{~min}$. The cells were then washed and fixed. The percentages of b) formazan-positive cells and c) cells that phagocytosed SOZ were analysed using spinning disc confocal microscopy. Data are presented as mean \pm SD. $n=3$. *: $p<0.05$, control versus wortmannin; **: $p<0.01$, M0 versus M0+MSC. d) Human blood-derived monocytes were cultured for 5 days, followed with stimulation by vehicle or $100 \mathrm{nM} \mathrm{PGE} 2$ for $24 \mathrm{~h}$. Levels of phospho-Akt (S473) and total Akt were analysed by Western blotting. Data are presented as mean $\pm S D . n=3$. ${ }^{*}: p<0.05$. e) Levels of $P_{G E}$ in culture supernatant of M0, MSCs and M0 exposed to MSCs in transwell were analysed by a PGE 2 ELISA kit. Data are presented as mean \pm SD. $n=3$. *: $p<0.05$. f) Human blood-derived monocytes were cultured for 5 days, then cocultured with MSCs in transwell for 3 days in the absence or presence of $10 \mu \mathrm{M} \mathrm{PGE}$ synthase inhibitor CAY10526. NBT assay was performed and the percentage of formazan-positive cells was quantified. Data are presented as mean \pm SD. $n=3$. ${ }^{* * *}$ : $p<0.001, M 0$ versus M0+MSC and control versus CAY10526.

To test whether $\mathrm{PGE}_{2}$ is required for the increase in the number of cells with enhanced phagosomal ROS, we inhibited $\mathrm{PGE}_{2}$ production with CAY10526, which prevented the increase in ROS-positive macrophages in response to MSC exposure (figure 5f). Thus, MSC production of $\mathrm{PGE}_{2}$ could lead to enhanced PI3K activation in a subset of macrophages and allow for enhanced (NOX2) NADPH oxidase assembly in response to phagocytosis, resulting in enhanced phagosomal ROS production.

\section{MSCs promote some features of M2-like macrophages}

Previous studies have identified that MSCs polarise a population of macrophages resembling an M2-like phenotype [29]. Our results support and extend these findings. Analysis of cell morphology revealed that some macrophage cells cultured with MSCs in transwell had an elongated appearance that is typical of many M2 cells (figure 6a). Quantification revealed that MSCs caused $\sim 20 \%$ of the population to adopt this morphology (figure 6b). An important functional characteristic of M2 cells is that, in contrast to M1 cells, they rapidly acidify newly formed phagosomes [15]. To determine if the MSC-induced "M2-like" cells behaved like bona fide $\mathrm{M} 2$ cells, we monitored phagosomal $\mathrm{pH}$ by ratio imaging in macrophage cells 
a)

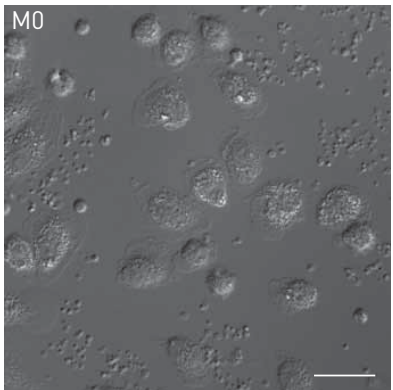

b) 60

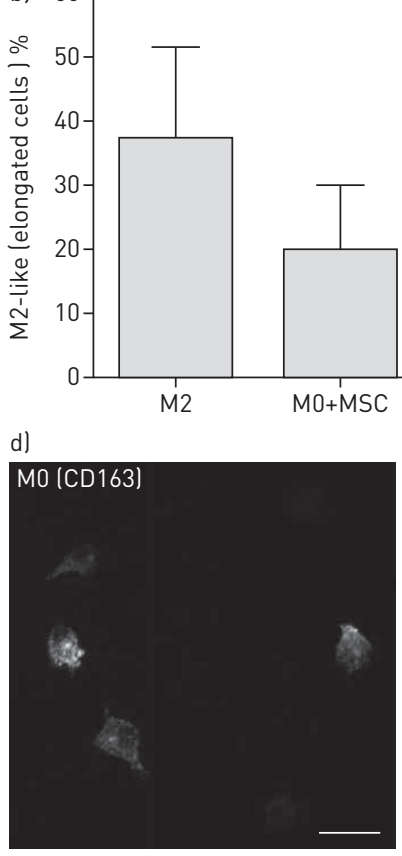

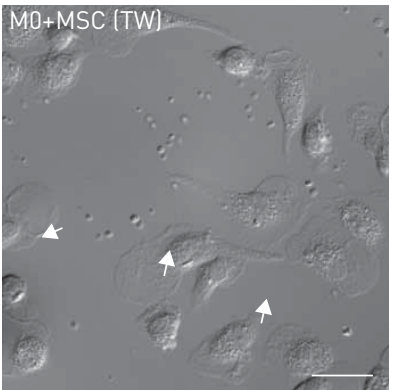
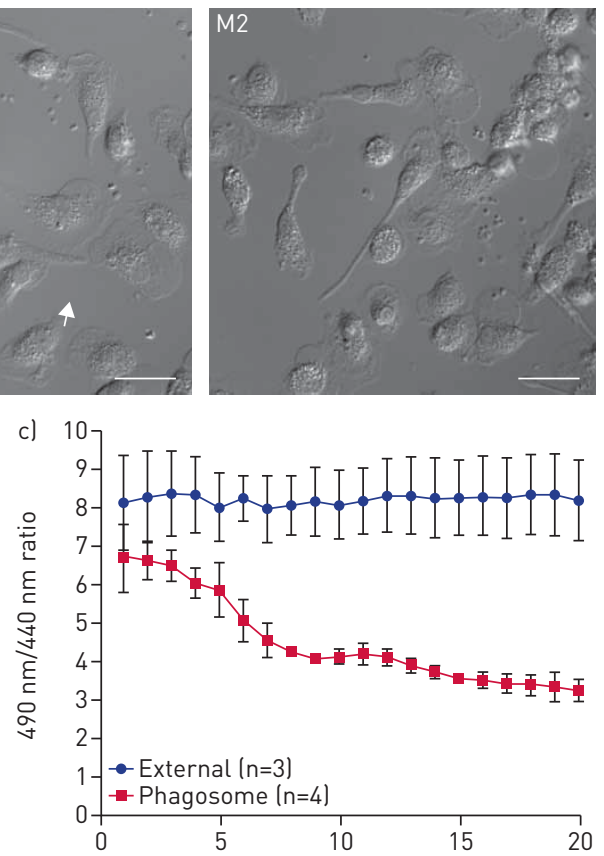

20 e)
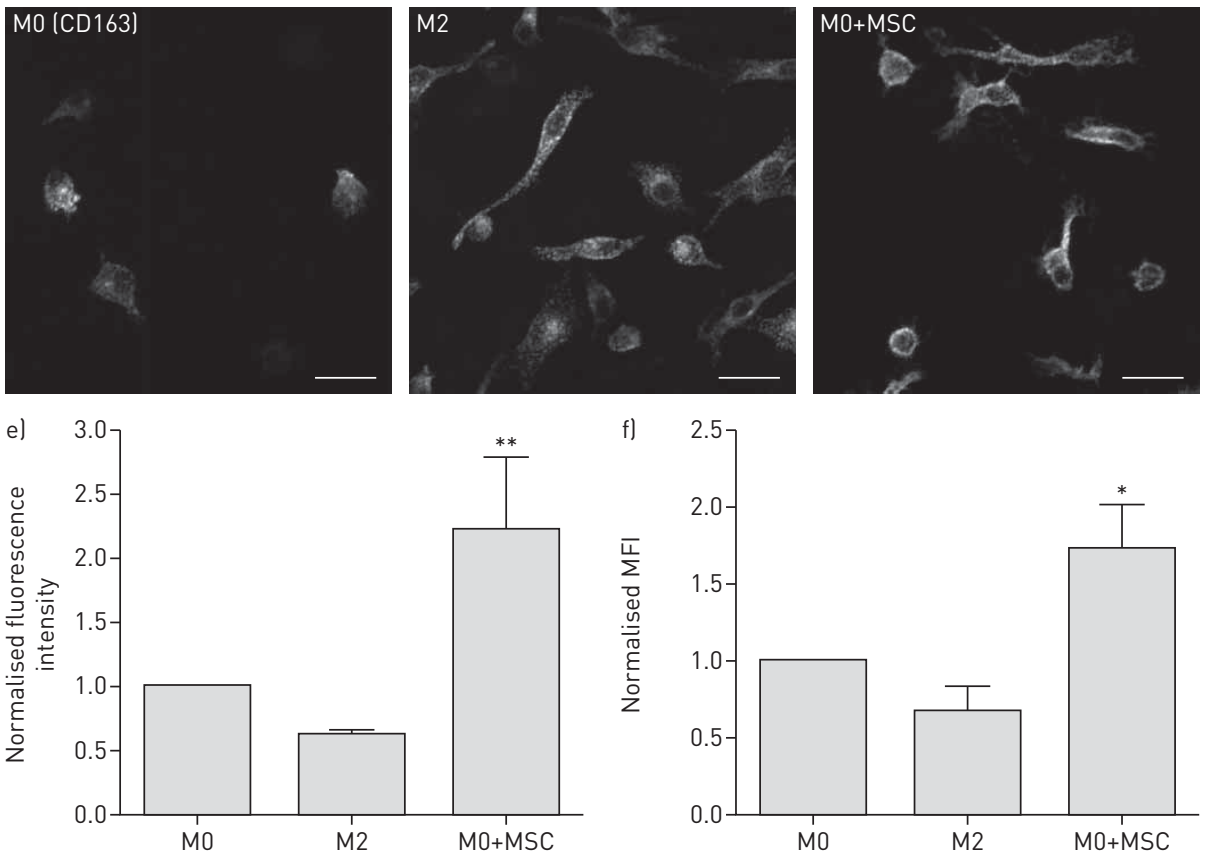

FIGURE 6 Mesenchymal stem/stromal cells (MSCs) cause some human macrophages to adopt an M2-like morphology and phenotype. a) Human blood-derived monocytes were cultured for 8 days with media containing serum (MO) or after 5 days the cells were cultured with bone marrow-derived MSCs in transwell (TW) for 3 days. M2 cells were generated as described in the Materials and methods. The cells were fixed and observed by differential interference contrast microscopy. Many M2 cells adopt an elongated morphology, which were evident in the M0+MSC condition (white arrows), but not in the M0 condition. Scale bar: $20 \mu \mathrm{m}$. b) The percentage of elongated cells was quantified. Data are presented as mean \pm SD. $n=7$ experiments. c) Human blood-derived monocytes were cultured for 5 days and then cultured with MSCs in TW for 3 days. Phagosomal $\mathrm{pH}$ was monitored in M2-like cells by $490 \mathrm{~nm} / 440 \mathrm{~nm}$ excitation wavelength ratio imaging using fluorescein isothiocyanate (FITC)-serum opsonised zymosan (SOZ) as outlined in the Materials and methods. In some experiments external nonphagocytosed FITC-SOZ particles were also imaged as a control. Data are presented as mean \pm SD. $n=3-4$ independent experiments. d) Macrophages were prepared as described in (a), and then fixed and immunostained for CD163. Representative confocal images. Scale bar: $20 \mu \mathrm{m}$. e) Cellular fluorescence intensity was measured using ImageJ (https://imagej.nih.gov/ij/) and values were normalised to the levels in $M 0$ cells. Data are presented as mean \pm SD. $n=3$ experiments. **: $p<0.01, M 2$ versus $M 0+M S C$. f) Flow cytometry analysis of CD163 under the various conditions. Mean fluorescence intensity (MFI) of the positive cell population. Data are presented as mean \pm SD. $n=4$. * $p<0.05, M 2$ versus M0+MSC. 
exposed to FITC-labelled SOZ. Elongated macrophage cells produced in response to MSC exposure rapidly acidified newly formed phagosomes as observed by the drop in the $490 \mathrm{~nm} / 444 \mathrm{~nm}$ excitation wavelength ratio (figure 6c), similar to M2 cells [15].

M2 macrophages are also distinguished by the expression of certain cell surface proteins [30, 31], such as CD163 in human cells $[31,32]$. We monitored localisation and levels of CD163 by immunofluorescence microscopy using a specific antibody [33]. The number of cells with CD163 immunostaining (both round and elongated) was higher in M0 cells exposed to MSCs compared with control M0 cells (figure 6d). Quantification of total fluorescence (figure 6e) and surface levels by flow cytometry (figure 6f) showed that MSC-treated macrophages express CD163 at levels higher than M0 cells or defined M2 cells.

Given that phagosomal ROS production is a feature of M1 macrophages, we determined whether M0 cells exposed to MSCs, particularly those with enhanced ROS production, had enhanced levels of M1-specific proteins such as CD40 [30]. Immunofluorescence microscopy confirmed CD40 surface staining in M1 macrophages that produce phagosomal superoxide as detected by the NBT assay (supplementary figure S3). However, macrophages exposed to MSCs had little or no CD40 staining (supplementary figure S3). Thus, although M1-like cells have some features of M1 cells, such as high phagosomal superoxide production, they lack markers of bona fide inflammatory M1 cells.

\section{MSCs enhance bacterial phagocytosis and killing in healthy human macrophages and in macrophages from patients with sepsis}

Given that the NADPH oxidase has an essential role in microbicidal capacity [27] and we observe an increase in cells with enhanced oxidase activity, we hypothesised that MSCs may promote bacterial killing via this mechanism. Phagocytosis of unopsonised E. coli (figure $7 \mathrm{a}-\mathrm{c}$ ) and B. cenocepacia (supplementary figure S4) was markedly increased in macrophage cells that had been exposed to MSCs either in transwell or coculture. We next examined the effect of MSCs on bacterial killing. As shown in figure 7d, macrophage cells that had been exposed to MSCs had greater capacity to kill E. coli compared with control M0 or M2 cells. Bacterial killing was markedly reduced by inhibition of the NADPH oxidase with DPI, suggesting that M1-like cells are likely primarily responsible for the enhanced bacterial killing observed.

Given the pre-clinical evidence for MSCs as a novel adjunct to antibiotic therapy, we also examined the effect of MSCs on monocyte-derived macrophages isolated from patients with sepsis. MSCs enhanced phagocytosis of SOZ and production of phagosomal ROS (figure 8a and b), as well as phagocytosis of E. coli (figure $8 \mathrm{c}$ and d). This suggests that MSCs could enhance macrophage phagocytosis and bacterial killing in sepsis.

\section{Discussion}

In this study we have uncovered the ability of bone marrow-derived MSCs to affect macrophage polarisation and function, in vitro and in vivo, to induce a heterogeneous population. Our studies implicate $\mathrm{PGE}_{2}$ as an important mediator of the effect (figure 9). To the best of our knowledge, we are the first to report such a striking heterogeneity in monocyte-derived macrophages exposed to MSCs. Unexpectedly, we found that MSCs induce coexistent M1-like and M2-like macrophage phenotypes. The fact that macrophages can be directed to a phenotype endowed with enhanced antimicrobial capacity (M1-like), and also an inflammation resolving and tissue repair phenotype (M2-like), may account for the paradoxical effect of these cells in pathogenic conditions such as sepsis in vivo.

Previous works in this area by KIм and Нематті [29] and others [14, 34, 35] have described a population of M2-like human macrophages generated in vitro after coculture with human MSCs, defined as CD206 ${ }^{+}$, IL-10 ${ }^{\text {high }}$, IL-12 $2^{\text {low }}$ IL- $6^{\text {high }}$ and tumour necrosis factor (TNF)- $\alpha^{\text {low }}$. Here, we find that the M2-like macrophage phenotype induced by MSCs in a fraction of the population indeed had many features associated with M2 macrophage cells, including elongated shape [36], surface markers $\left(\mathrm{CD} 163^{+}\right)$, acute phagosomal acidification and low NOX2 expression with limited phagosomal superoxide production [15].

Remarkably, MSCs also induced an M1-like macrophage phenotype in a portion of the macrophage population, characterised by enhanced phagosomal superoxide production. However, unlike defined M1 macrophages, MSC-induced M1-like cells have low or undetectable levels of the M1 macrophage marker CD40. Importantly, this M1-like phenotype was also observed in isolated alveolar macrophages from an in vivo rodent model of $E$. coli pneumonia in response to i.v. MSC treatment.

We found that MSCs induced a PI3K- and $\mathrm{PGE}_{2}$-dependent increase in NADPH oxidase activity. We also demonstrated an enhanced ability to kill E. coli in macrophages exposed to MSCs, which was significantly impaired by inhibition of the NADPH oxidase. It is well established that activation of the phagocyte NADPH oxidase (NOX2) is a vital innate immune mechanism [27]. Indeed, many intracellular pathogens known to thrive in the acidic phagolysosome, such as Leishmania spp., are particularly sensitive to 
a)
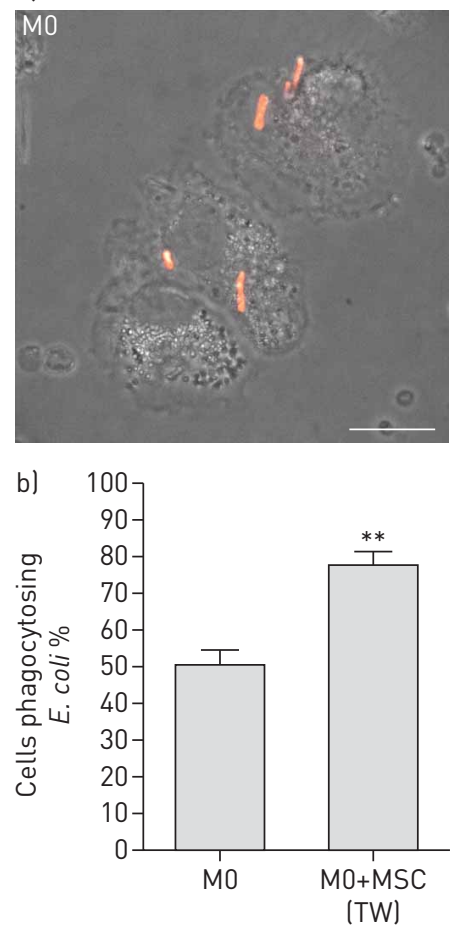

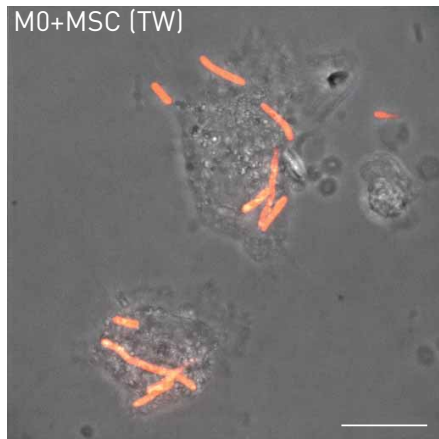

c)

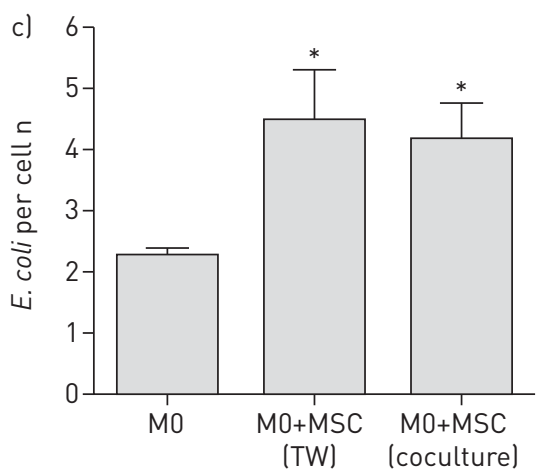

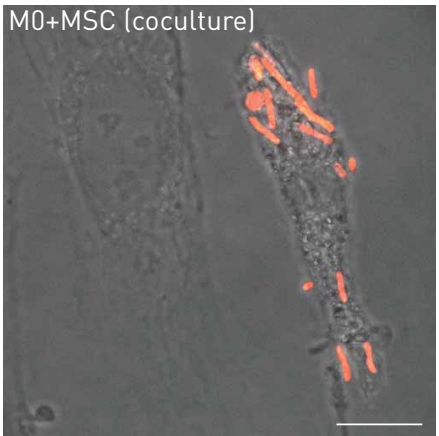

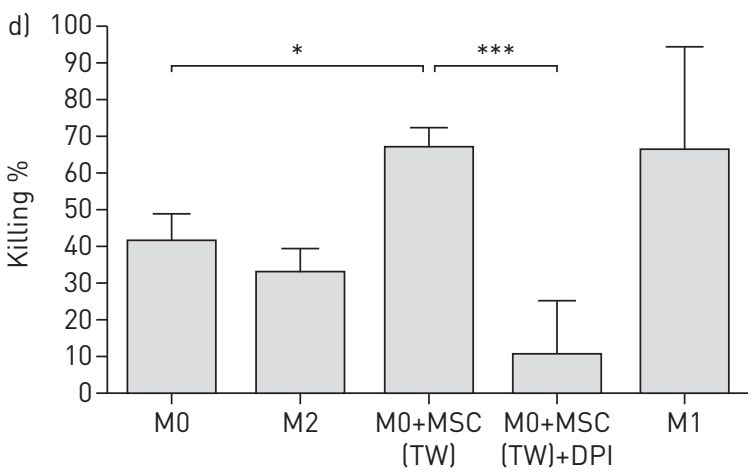

FIGURE 7 Mesenchymal stem/stromal cell (MSC) exposure enhances Escherichia coli phagocytosis and killing. a) Human blood-derived monocytes were cultured for 8 days with media containing serum (MO) or after 5 days the cells were cultured with bone marrow-derived MSCs in transwell (TW) or coculture for 3 days. The cells were then incubated with live Red Fluorescent Protein (RFP)-expressing E. coli that were spun down onto the macrophage cells. After 20 min the cells were washed with PBS, fixed, and then imaged by light and fluorescence microscopy. Representative extended focus images of combined phase contrast and RFP fluorescence. Scale bar: $10 \mu \mathrm{m}$. b) The percentage of cells that phagocytosed bacteria and $c$ ) the number of $E$. coli cells phagocytosed or bound per macrophage cell. Data are presented as mean \pm SE. $n=4-5$ independent experiments. *: p<0.05; **: p<0.01, M0 versus M0+MSC (TW or coculture). d) Macrophage cells were prepared as described in (a), and assessment of phagocytic E. coli killing was performed as outlined in the Materials and methods. Data are presented as mean \pm SE. $n=6$ independent experiments. ${ }^{*} p<0.05$, M0 versus M0+MSC (TW); ${ }^{* * *}$ : $p<0.001, M 0+M S C$ (TW) versus $M 0+M S C$ (TW)+diphenyleneiodonium (DPI)

ROS-mediated killing [37, 38]. The NADPH oxidase catalyses the formation of the highly unstable superoxide anion, which can be converted to hydrogen peroxide. Both of these ROS can damage proteins, lipids and DNA, resulting in microbicidal activity [39]. Our data support a key role for augmented NADPH oxidase (NOX2) induced by MSCs in MSC-induced enhanced bacterial killing. MSCs also enhance phagocytosis and phagosomal ROS generation in macrophages obtained from patients with severe sepsis. Monocyte anergy and immune suppression contribute to an increased risk of adverse events in sepsis [40]. Restoration of monocyte/macrophage antimicrobial function with MSCs represents a promising therapeutic strategy. Our findings provide strong support that MSCs can indeed promote such an improved microbial clearance. We suggest that augmented phagocytosis and oxidase-mediated killing could be one of the mechanisms for the beneficial therapeutic effects of MSCs.

ROS play a critical role in a multitude of cellular processes, including destruction of phagocytosed bacteria [41]. Given the novel M1-like phenotype uncovered, we focused our efforts on identifying how MSCs might mediate enhanced phagosomal ROS production in macrophages. The phagocytic NADPH oxidase complex consists of membrane-bound subunits (gp91 phox and $\mathrm{p} 22^{\text {phox }}$ (NOX2)) and an array of cytoplasmic factors $\left(\mathrm{p} 40^{\text {phox }}, \mathrm{p} 47^{\text {phox }}, \mathrm{p} 67^{\text {phox }}\right.$ and $\mathrm{Rac}$ ) that translocate to the membrane, resulting in formation of a primed and active NADPH oxidase complex. We found that oxidase assembly is increased in macrophages with enhanced ability to produce phagosomal superoxide, as monitored by the distribution of $\mathrm{p} 47^{\text {phox }}$ in the NBT assay, and that PI3K activity is essential for the increase in M1-like cells. Basal PI3K activity was enhanced in the macrophage population exposed to MSCs. Presumably this would enhance the ability to recruit cytosolic oxidase components in a subset of macrophages in response to phagocytosis, which is dependent on the production of phosphoinositides to facilitate the recruitment of cytosolic oxidase subunits to the membrane-bound subunits at the forming phagosome [26-28].

NOX2 activation by MSCs in our study is $\mathrm{PGE}_{2}$ dependent. However, prostanoids, including $\mathrm{PGE}_{2}$, have traditionally been believed to exert anti-inflammatory effects in macrophages. $\mathrm{PGE}_{2}$ has been 
a)
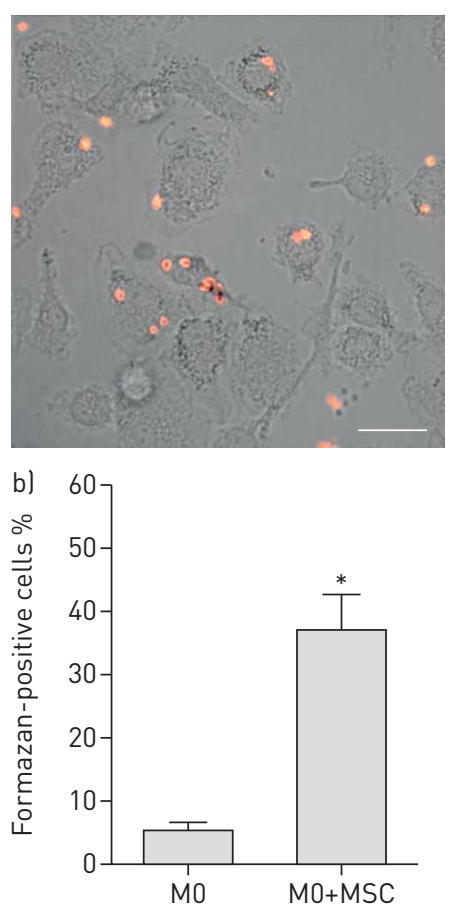

(TW)

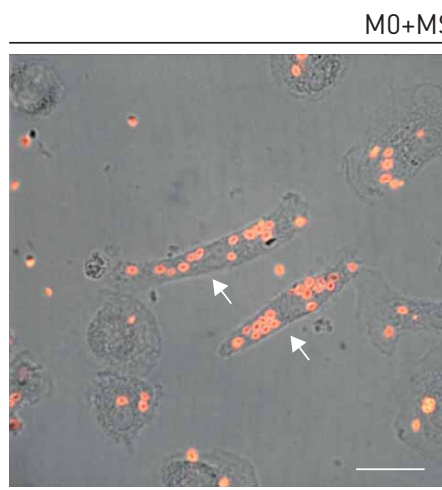

c)

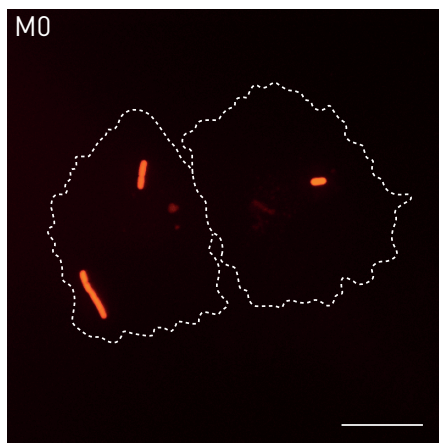

MO+MSC (TW)

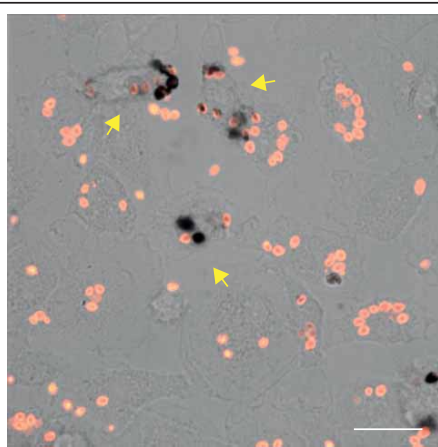

\section{$\mathrm{M} 0+\mathrm{MSC}(\mathrm{TW})$}

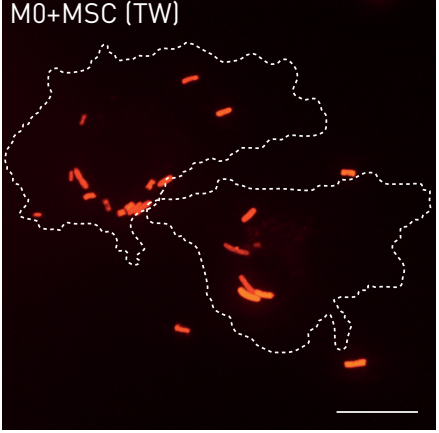

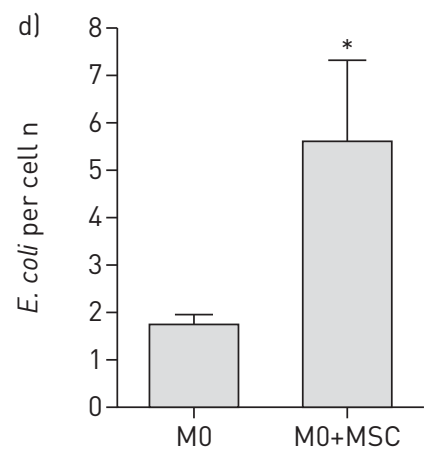

(TW)

FIGURE 8 Mesenchymal stem/stromal cells (MSCs) induce M1-like and M2-like phenotypes and enhance phagocytosis in macrophages derived from patients with sepsis. a) Human blood-derived monocytes were isolated from patients with sepsis and cultured for 8 days with media containing serum (M0) or after 5 days the cells were cultured with bone marrow-derived MSCs in transwell (TW) for 3 days. A serum opsonised zymosan-nitroblue tetrazolium assay was performed as in figure 1a. The cells were fixed and imaged by confocal microscopy. Both M2-like cells (white arrows) and M1-like cells with detectable phagosomal formazan (yellow arrows) were observed. Result is representative of $\mathrm{n}=4$ patients. Scale bar: $20 \mu \mathrm{m}$. b) Quantification of phagosomal formazan-positive cells. Data are presented as mean \pm SD. $n=4$. ${ }^{*}: p<0.05$. c) Cells were prepared as in (a), and E. coli phagocytosis was monitored as described in the Materials and methods. MSC-exposed macrophages had increased levels of intracellular or bound E. coli. Representative images. Scale bar: $10 \mu \mathrm{m}$. d) The number of E. coli cells phagocytosed or bound per macrophage cell was quantified. Data are presented as mean \pm SD. $n=3$ patients. *: $p<0.05$.

demonstrated to inhibit the production of pro-inflammatory molecules such as TNF- $\alpha$ [42] and increase the secretion of anti-inflammatory cytokines such as IL-10 [43]. Regarding the MSC-mediated polarisation of macrophages to an M2 phenotype, a major role has been ascribed to $\mathrm{PGE}_{2}$ [14]. However, studies of prostanoid receptors in mice, on T-helper (Th) cells and dendritic cells, revealed that different prostanoid receptors regulate the immune response at various steps by exerting often opposing actions [44, 45]. In particular, although $\mathrm{PGE}_{2}$ suppresses pro-inflammatory actions of macrophages and Th1 differentiation by raising intracellular cyclic AMP concentrations, $\mathrm{PGE}_{2}$ also facilitates Th1 differentiation via PI3K. Moreover, $\mathrm{PGE}_{2}$ induces production of ROS in mast cells in a PI3K-dependent manner [46]. Here, we demonstrated that inhibition of MSC-derived $\mathrm{PGE}_{2}$ prevented MSC-induced phagosomal ROS production, while coculture of MSCs and macrophages induced enhanced production of $\mathrm{PGE}_{2}$ from MSCs. PGE alone activated PI3K and ROS production in macrophages. Diverse functions of $\mathrm{PGE}_{2}$ may be dependent on several factors, including differential expression of E-prostanoid (EP) receptors on macrophages [47], dynamics of EP receptor expression during the course of macrophage activation [48], differences in ligand affinity of EP receptors and kinetics of receptor desensitisation, and finally the specific downstream pathways of each receptor [49].

It is also important to point out that although the NADPH oxidase is a key component of the antibacterial response, other factors may also contribute to enhanced bacterial killing. Indeed, while M2 cells have limited NOX2 expression they were still able to kill E. coli, albeit less efficiently than M1 or M1-like cells. Presumably, this is due to the degradative capacity in the low $\mathrm{pH}$ environment of the phagolysosome [50]. Furthermore, mitochondrial ROS has also been implicated in contributing to pathogen killing [51], and recent studies have shown that MSCs can potentially deliver mitochondria to macrophage cells via extracellular vesicles [52] and via tunnelling nanotubules [22].

How do MSCs produce different macrophage phenotypes from the same blood-derived monocytes? At least three distinct types of blood-derived monocytes exist [53] and presumably at least one of these 


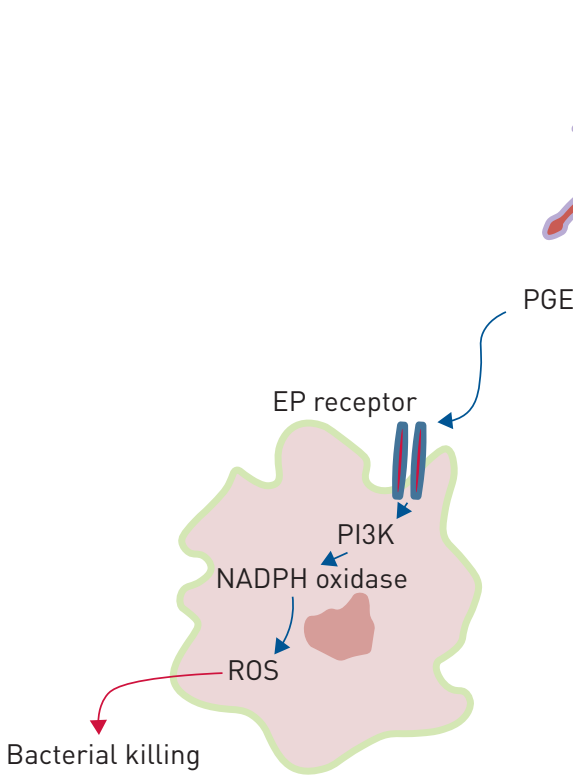

M1-like macrophage

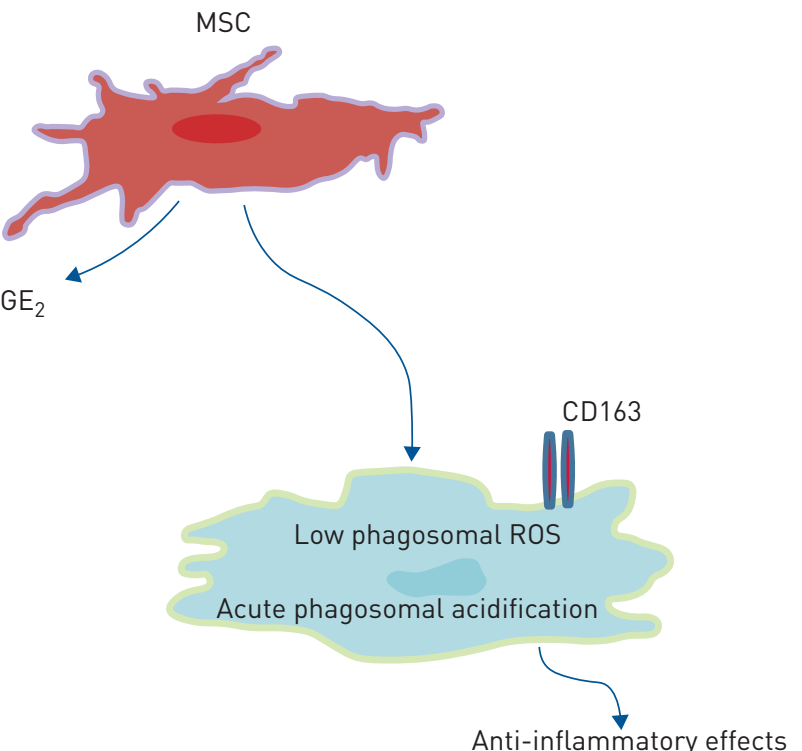

M2-like macrophage

FIGURE 9 Schematic showing the mechanisms by which mesenchymal stem/stromal cells (MSCs) alter macrophage function. $\mathrm{PGE}_{2}$ : prostaglandin $\mathrm{E}_{2}$; EP: E-prostanoid; PI3K: phosphatidylinositol 3-kinase; ROS: reactive oxygen species.

populations gives rise to macrophages that respond to $\mathrm{PGE}_{2}$ to produce the M1-like phenotype we have observed. As we, and others, have shown, MSCs also induce an M2-like phenotype from monocytes [12, 14]. This directed maturation is partially regulated via direct cell contact, but also by MSC secretion of $\mathrm{PGE}_{2}$, IL-6, indoleamine 2,3-dioxygenase and TNF-stimulated gene 6 [14, 34, 35, 54]. However, other factors may also contribute to MSC-induced effects. Other investigators have recently demonstrated that macrophages engulf mitochondria-containing microvesicles and microRNA-containing exosomes from MSCs, thereby enhancing macrophage bioenergetics while inhibiting macrophage activation [23, 52]. Lee and colleagues have demonstrated some antimicrobial effects of MSC-secreted keratinocyte growth factor and microvesicles on human monocytes $[55,56]$. What is clear from these and our own studies is that MSC immune modulation of monocytes and macrophages is complex, likely not accomplished by a single secreted factor, and represents a balance of pro- and anti-inflammatory stimulation.

There are a number of limitations to these experiments. MSCs modulate macrophage function and bacterial killing by a variety of mechanisms not examined in these studies, including transfer of mitochondria and regulatory microRNAs [52], and secretion of antimicrobial peptides [24]. We focused on $\mathrm{PGE}_{2}$, as it has an important role in MSC effects in sepsis, as well as macrophage polarisation [14]. We did not augment $\mathrm{PGE}_{2}$ secretion in our studies, which could serve as a mechanism to enhance MSC potency, although this approach is likely to have off-target effects. The timing of the effect also needs further evaluation: our in vitro experiments involved 48-72 h exposure of macrophages to MSCs, which may not reflect in vivo MSC-macrophage interactions. Although our studies examined the effects of MSCs on macrophages from healthy volunteers, as well as septic patients, we did not isolate macrophages from critically ill patients without sepsis. It is likely, however, that MSCs alter phagocytosis and ROS generation in a similar manner to healthy controls. Finally, future studies using single-cell RNA sequencing and bioinformatics approaches could provide a more detailed characterisation of the heterogeneous macrophage populations we observed.

The future of MSC therapy depends on the development of a potent, consistent cell-based therapeutic. While cell-free approaches, using MSC-derived extracellular vesicles, have emerged as a promising treatment strategy, we observed a synergy and cross-talk between MSCs and macrophages, which points to the need for the cell itself to achieve maximal therapeutic effect. Factors limiting the potential of MSCs as a therapy for sepsis include limited MSC persistence, insufficient and variable immunomodulatory potency, and dependence on in vivo activation by host inflammatory mediators [57]. A detailed understanding of the host response to MSCs, as in this study and others [22, 52], is necessary in order to develop a consistent therapeutic and to identify patients most likely to benefit. Previous clinical trials of 
MSCs for other disease indications demonstrate the need to develop in vitro and in vivo models that can predict successful host response to MSC therapy [58]. Our study identifies phagocytosis and ROS production as host factors during sepsis that could be rescued by MSC treatment.

Acknowledgements: We would like to thank Chris Spring (St Michael's Hospital, Toronto, ON, Canada) for assistance with flow cytometry analysis, and John E. Davies and Tissue Regeneration Therapeutics (Toronto, ON, Canada) for providing umbilical cord mesenchymal stem cells.

Support statement: G.F. Curley and J.G. Laffey are funded by the Canadian Institutes of Health Research (CIHR-312714) and hold a Merit Award and a Clinician Scientist Transition Award, respectively, from the Dept of Anesthesia, University of Toronto. G.F. Curley holds an award from the International Anesthesia Research Society and is funded by a Government of Ontario, Ministry of Research and Innovation, Early Researcher Award. G.F. Curley and K. Szaszi were funded by an award from St Michael's Hospital Translational Innovation Fund. K.C. Tatham is funded by the National Institute of Health Research (UK), the European Institute of Innovation and Technology, and Academy of Medical Sciences (UK). Funding information for this article has been deposited with the Crossref Funder Registry.

Conflict of interest: G.F. Curley reports grants paid to his institution from the Government of Ontario, Ministry of Research and Innovation (Early Researcher Award), Canadian Institute of Health Research (Operating Grant CIHR-312714) and St Michael's Hospital Translational Innovation Fund (TIF Award Winner 2015), during the conduct of the study. J.G. Laffey reports grants from the Canadian Institutes for Health Research and salary support from the Dept of Anesthesia, University of Toronto, during the conduct of the study.

\section{References}

1 Brun-Buisson C, Meshaka P, Pinton P, et al. EPISEPSIS: a reappraisal of the epidemiology and outcome of severe sepsis in French intensive care units. Intensive Care Med 2004; 30: 580-588.

2 Liu V, Escobar GJ, Greene JD, et al. Hospital deaths in patients with sepsis from 2 independent cohorts. JAMA 2014; 312: 90-92.

3 Mendez-Ferrer S, Michurina TV, Ferraro F, et al. Mesenchymal and haematopoietic stem cells form a unique bone marrow niche. Nature 2010; 466: 829-834.

4 Shi C, Jia T, Mendez-Ferrer S, et al. Bone marrow mesenchymal stem and progenitor cells induce monocyte emigration in response to circulating Toll-like receptor ligands. Immunity 2011; 34: 590-601.

5 Friedenstein AJ, Petrakova KV, Kurolesova AI, et al. Heterotopic of bone marrow. Analysis of precursor cells for osteogenic and hematopoietic tissues. Transplantation 1968; 6: 230-247.

6 Crisan M, Yap S, Casteilla L, et al. A perivascular origin for mesenchymal stem cells in multiple human organs. Cell Stem Cell 2008; 3: 301-313.

7 Curley GF, Ansari B, Hayes M, et al. Effects of intratracheal mesenchymal stromal cell therapy during recovery and resolution after ventilator-induced lung injury. Anesthesiology 2013; 118: 924-932.

8 Curley GF, Hayes M, Ansari B, et al. Mesenchymal stem cells enhance recovery and repair following ventilator-induced lung injury in the rat. Thorax 2012; 67: 496-501.

9 Curley GF, Jerkic M, Dixon S, et al. Cryopreserved, xeno-free human umbilical cord mesenchymal stromal cells reduce lung injury severity and bacterial burden in rodent Escherichia coli-induced acute respiratory distress syndrome. Crit Care Med 2017; 45: e202-e212.

10 McIntyre LA, Stewart DJ, Mei SHJ, et al. Cellular immunotherapy for septic shock. A phase I clinical trial. Am J Respir Crit Care Med 2018; 197: 337-347.

11 Liu KD, Wilson JG, Zhuo H, et al. Design and implementation of the START (STem cells for ARDS Treatment) trial, a phase $1 / 2$ trial of human mesenchymal stem/stromal cells for the treatment of moderate-severe acute respiratory distress syndrome. Ann Intensive Care 2014; 4: 22.

$12 \mathrm{Ko} J \mathrm{H}$, Lee HJ, Jeong HJ, et al. Mesenchymal stem/stromal cells precondition lung monocytes/macrophages to produce tolerance against allo- and autoimmunity in the eye. Proc Natl Acad Sci USA 2016; 113: 158-163.

13 Vasandan AB, Jahnavi S, Shashank $\mathrm{C}$, et al. Human mesenchymal stem cells program macrophage plasticity by altering their metabolic status via a $\mathrm{PGE}_{2}$-dependent mechanism. Sci Rep 2016; 6: 38308.

14 Nemeth K, Leelahavanichkul A, Yuen PST, et al. Bone marrow stromal cells attenuate sepsis via prostaglandin $\mathrm{E}_{2}$-dependent reprogramming of host macrophages to increase their interleukin-10 production. Nat Med 2009; 15: $42-49$.

15 Canton J, Khezri R, Glogauer M, et al. Contrasting phagosome pH regulation and maturation in human M1 and M2 macrophages. Mol Biol Cell 2014; 25: 3330-3341.

16 Miles SA, Conrad SM, Alves RG, et al. A role for IgG immune complexes during infection with the intracellular pathogen Leishmania. J Exp Med 2005; 201: 747-754.

17 Muller U, Stenzel W, Kohler G, et al. IL-13 induces disease-promoting type 2 cytokines, alternatively activated macrophages and allergic inflammation during pulmonary infection of mice with Cryptococcus neoformans. $J$ Immunol 2007; 179: 5367-5377.

18 Devaney J, Horie S, Masterson C, et al. Human mesenchymal stromal cells decrease the severity of acute lung injury induced by E. coli in the rat. Thorax 2015; 70: 625-635.

19 Krasnodembskaya A, Samarani G, Song Y, et al. Human mesenchymal stem cells reduce mortality and bacteremia in Gram-negative sepsis in mice in part by enhancing the phagocytic activity of blood monocytes. Am J Physiol Lung Cell Mol Physiol 2012; 302: L1003-L1013.

20 Mei SH, Haitsma JJ, Dos Santos CC, et al. Mesenchymal stem cells reduce inflammation while enhancing bacterial clearance and improving survival in sepsis. Am J Respir Crit Care Med 2010; 182: 1047-1057.

21 O'Croinin DF, Nichol AD, Hopkins N, et al. Sustained hypercapnic acidosis during pulmonary infection increases bacterial load and worsens lung injury. Crit Care Med 2008; 36: 2128-2135.

22 Jackson MV, Morrison TJ, Doherty DF, et al. Mitochondrial transfer via tunneling nanotubes (TNT) is an important mechanism by which mesenchymal stem cells enhance macrophage phagocytosis in the in vitro and in vivo models of ARDS. Stem Cells 2016; 34: 2210-2223. 
23 Morrison TJ, Jackson MV, Cunningham EK, et al. Mesenchymal stromal cells modulate macrophages in clinically relevant lung injury models by extracellular vesicle mitochondrial transfer. Am J Respir Crit Care Med 2017; 196: $1275-1286$

24 Krasnodembskaya A, Song Y, Fang X, et al. Antibacterial effect of human mesenchymal stem cells is mediated in part from secretion of the antimicrobial peptide LL-37. Stem Cells 2010; 28: 2229-2238.

25 Gupta N, Krasnodembskaya A, Kapetanaki M, et al. Mesenchymal stem cells enhance survival and bacterial clearance in murine Escherichia coli pneumonia. Thorax 2012; 67: 533-539.

26 Leto TL, Morand S, Hurt D, et al. Targeting and regulation of reactive oxygen species generation by Nox family NADPH oxidases. Antioxid Redox Signal 2009; 11: 2607-2619.

27 Nunes P, Demaurex N, Dinauer MC. Regulation of the NADPH oxidase and associated ion fluxes during phagocytosis. Traffic 2013; 14: 1118-1131.

28 Vieira OV, Botelho RJ, Rameh L, et al. Distinct roles of class I and class III phosphatidylinositol 3-kinases in phagosome formation and maturation. J Cell Biol 2001; 155: 19-25.

29 Kim J, Hematti P. Mesenchymal stem cell-educated macrophages: a novel type of alternatively activated macrophages. Exp Hematol 2009; 37: 1445-1453.

30 Murray PJ, Allen JE, Biswas SK, et al. Macrophage activation and polarization: nomenclature and experimental guidelines. Immunity 2014; 41: 14-20.

31 Roszer T. Understanding the mysterious M2 macrophage through activation markers and effector mechanisms. Mediators Inflamm 2015; 2015: 816460.

32 Jaguin M, Houlbert N, Fardel O, et al. Polarization profiles of human M-CSF-generated macrophages and comparison of M1-markers in classically activated macrophages from GM-CSF and M-CSF origin. Cell Immunol 2013; 281: 51-61.

33 Fuentes-Duculan J, Suarez-Farinas M, Zaba LC, et al. A subpopulation of CD163-positive macrophages is classically activated in psoriasis. J Invest Dermatol 2010; 130: 2412-2422.

34 Melief SM, Schrama E, Brugman MH, et al. Multipotent stromal cells induce human regulatory T cells through a novel pathway involving skewing of monocytes toward anti-inflammatory macrophages. Stem Cells 2013; 31: 1980-1991.

35 Francois M, Romieu-Mourez R, Li M, et al. Human MSC suppression correlates with cytokine induction of indoleamine 2,3-dioxygenase and bystander M2 macrophage differentiation. Mol Ther 2012; 20: 187-195.

36 McWhorter FY, Wang T, Nguyen P, et al. Modulation of macrophage phenotype by cell shape. Proc Natl Acad Sci USA 2013; 110: 17253-17258.

37 Novais FO, Nguyen BT, Beiting DP, et al. Human classical monocytes control the intracellular stage of Leishmania braziliensis by reactive oxygen species. J Infect Dis 2014; 209: 1288-1296.

38 Novais FO, Santiago RC, Bafica A, et al. Neutrophils and macrophages cooperate in host resistance against Leishmania braziliensis infection. J Immunol 2009; 183: 8088-8098.

39 Lam GY, Huang J, Brumell JH. The many roles of NOX2 NADPH oxidase-derived ROS in immunity. Semin Immunopathol 2010; 32: 415-430.

40 Delano MJ, Ward PA. Sepsis-induced immune dysfunction: can immune therapies reduce mortality? J Clin Invest 2016; 126: 23-31.

41 DeLeo FR, Allen LA, Apicella M, et al. NADPH oxidase activation and assembly during phagocytosis. J Immunol 1999; 163: 6732-6740.

42 Ratcliffe MJ, Walding A, Shelton PA, et al. Activation of E-prostanoid4 and E-prostanoid2 receptors inhibits TNF-alpha release from human alveolar macrophages. Eur Respir J 2007; 29: 986-994.

43 Menard G, Turmel V, Bissonnette EY. Serotonin modulates the cytokine network in the lung: involvement of prostaglandin $\mathrm{E}_{2}$. Clin Exp Immunol 2007; 150: 340-348.

44 Kabashima K, Sakata D, Nagamachi $\mathrm{M}$, et al. Prostaglandin $\mathrm{E}_{2}-\mathrm{EP} 4$ signaling initiates skin immune responses by promoting migration and maturation of Langerhans cells. Nat Med 2003; 9: 744-749.

45 Yao C, Sakata D, Esaki Y, et al. Prostaglandin $\mathrm{E}_{2}-\mathrm{EP} 4$ signaling promotes immune inflammation through $\mathrm{T}_{\mathrm{H}} 1$ cell differentiation and $\mathrm{T}_{\mathrm{H}} 17$ cell expansion. Nat Med 2009; 15: 633-640.

46 Kuehn HS, Jung MY, Beaven MA, et al. Prostaglandin $\mathrm{E}_{2}$ activates and utilizes mTORC2 as a central signaling locus for the regulation of mast cell chemotaxis and mediator release. J Biol Chem 2011; 286: 391-402.

47 Sokolowska M, Chen LY, Liu Y, et al. Prostaglandin $\mathrm{E}_{2}$ inhibits NLRP3 inflammasome activation through EP4 receptor and intracellular cyclic AMP in human macrophages. J Immunol 2015; 194: 5472-5487.

48 Hubbard NE, Lee S, Lim D, et al. Differential mRNA expression of prostaglandin receptor subtypes in macrophage activation. Prostaglandins Leukot Essent Fatty Acids 2001; 65: 287-294.

49 Kalinski P. Regulation of immune responses by prostaglandin $\mathrm{E}_{2}$. J Immunol 2012; 188: 21-28.

50 Ip WK, Sokolovska A, Charriere GM, et al. Phagocytosis and phagosome acidification are required for pathogen processing and MyD88-dependent responses to Staphylococcus aureus. J Immunol 2010; 184: 7071-7081.

51 West AP, Brodsky IE, Rahner C, et al. TLR signaling augments macrophage bactericidal activity through mitochondrial ROS. Nature 2011; 472: 476-480.

52 Phinney DG, Di Giuseppe M, Njah J, et al. Mesenchymal stem cells use extracellular vesicles to outsource mitophagy and shuttle microRNAs. Nat Commun 2015; 6: 8472 .

53 Murray PJ, Wynn TA. Protective and pathogenic functions of macrophage subsets. Nat Rev Immunol 2011; 11: 723-737.

54 Mittal M, Tiruppathi C, Nepal S, et al. TNFalpha-stimulated gene-6 (TSG6) activates macrophage phenotype transition to prevent inflammatory lung injury. Proc Natl Acad Sci USA 2016; 113: E8151-E8158.

55 Lee JW, Krasnodembskaya A, McKenna DH, et al. Therapeutic effects of human mesenchymal stem cells in ex vivo human lungs injured with live bacteria. Am J Respir Crit Care Med 2013; 187: 751-760.

56 Monsel A, Zhu YG, Gennai S, et al. Therapeutic effects of human mesenchymal stem cell-derived microvesicles in severe pneumonia in mice. Am J Respir Crit Care Med 2015; 192: 324-336.

57 Galipeau J. The mesenchymal stromal cells dilemma - does a negative phase III trial of random donor mesenchymal stromal cells in steroid-resistant graft-versus-host disease represent a death knell or a bump in the road? Cytotherapy 2013; 15: 2-8.

58 Trounson A, McDonald C. Stem cell therapies in clinical trials: progress and challenges. Cell Stem Cell 2015; 17: $11-22$. 